\title{
Impact assessment of mass gatherings using labelling procedure in ED, Nouvelle-Aquitaine, 2016
}

\author{
Laure Meurice ${ }^{\star 1}$, Anne Bernadou ${ }^{1}$, Antoine Tignon², Patricia Siguret ${ }^{2}$, \\ Stéphanie Vandentorren ${ }^{1}$, Céline Caserio-Schönemann ${ }^{3}$, Laurent Maillard² and \\ caroline Ligier ${ }^{2}$
}

${ }^{1}$ French institute for Public Health Surveillance, Nouvelle-Aquitaine Regional Office, Bordeaux, France, Bordeaux, France; ${ }^{2}$ Regional Emergency Observatory Nouvelle-Aquitaine, Bordeaux, France; ${ }^{3}$ French institute for Public Health Surveillance, Saint Maurice,

France

\section{Objective}

To access the potential health impact on the population during mass gathering over time using labelling procedure in emergency department (ED).

\section{Introduction}

The massive flow of people to mass gathering events, such as festivals or sports events like EURO 2016, may increase public health risks. In the particular context of several terrorist attacks that took place in France in 2015, the French national Public Health agency has decided to strengthen the population health surveillance systems using the mandatory notification disease system and the French national syndromic surveillance SurSaUD®.

The objectives in terms of health surveillance of mass gathering are: $1 /$ the timely detection of a health event (infectious cluster, environmental exposure, collective foodborne disease...) $2 /$ the health impact assessment of an unexpected event such as a terrorist attack.

In collaboration with the Regional Emergency Observatory (ORU), a procedure for the labeling of emergencies has been tested to identify the ED records that could be considered as linked to the event.

\section{Methods}

During summer 2016, the procedure was tested on seven major festive events throughout the region. In addition to the main medical diagnosis, a specific ICD-10 code "Y3388" was chosen to be used in associated diagnosis for records that were supposed to linked to the event.

Information on the labeling procedure was insured by the ORU to the emergency departments.

All records with medical diagnoses or medical pattern beginning by Y33 have been analyzed.

\section{Results}

No significant increase in the global indicator was observed in the ED impacted by mass gathering. The ED labelling procedure identified 260 records: two thirds corresponded to young men and $17 \%$ came from abroad. Among the 250 records labeled in associated diagnosis, $39 \%$ were associated to traumatisms and $31 \%$ corresponded to alcohol intake.

\section{Conclusions}

This study shows that a labelling procedure allows the identification, quantification and characterization of the population ED records associated with mass gathering. Additionally, a labelling procedure to assess a potential impact of an event as mass gathering can be implemented fairly rapidly.
Table 1 - Main diagnoses identified among the labelled passages Y33 (88) in associated diagnosis $(\mathrm{n}=250)$

\begin{tabular}{|c|c|c|}
\hline Main diagnosis & Number of records labeled Y33 & Proportion among the records labeled in associated diagnoses \\
\hline Traumatisms & 98 & $39,2 \%$ \\
\hline Alcohol & 78 & $31,2 \%$ \\
\hline Malaise & 5 & $2,0 \%$ \\
\hline Other & 69 & $27,6 \%$ \\
\hline
\end{tabular}

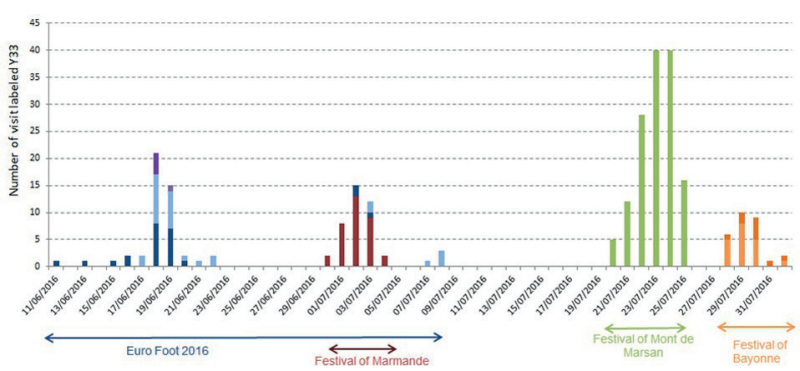

\section{Keywords}

impact assessment; mass gathering; emergency data; labelling procedure; labelling procedure

\section{Acknowledgments}

To the emergency departments and the Emergency Observatory Regional network.

\section{*Laure Meurice}

E-mail: laure.meurice@santepubliquefrance.fr 